\title{
LE SUPPLICHE DEI MUSICISTI DELLA CAPPELLA MUSICALE DEL SANTO DI PADOVA, (SEC. XVIII) RIORDINAMENTO ARCHIVISTICO E ALCUNI PERCORSI DI RICERCA NELL'ARCHIVIO DELLA VENERANDA ARCA DI SANT'ANTONIO
}

\author{
GIULIA FOLADORE \\ Associazione Centro Studi Antoniani, Padova
}

Izvleček: Prispevek govori o novejših preureditvah arhivskega gradiva in opisni analizi prošenj glasbenikov iz 18. stoletja, ki so ohranjene varhivu Veneranda Arca di Sant'Antonio v Padovi. Opisani so kriteriji popisa in uporabljeni metodološki principi ter nakazane možne nove smeri raziskav.

\begin{abstract}
The article provides information about the recent archival reorganization and descriptive analysis of the petitions by eighteenth-century musicians preserved in the archive of the Veneranda Arca di Sant'Antonio di Padova, explaining the criteria adopted and methodological principles applied, and outlining possible new avenues of research.
\end{abstract}

Ključne besede: bazilika Il Santo v Padovi, Veneranda Arca di Sant'Antonio, zgodovinski arhivi, prošnje glasbenikov, glasbena kapela.

Keywords: the Basilica of the Santo in Padua, Veneranda Arca di Sant'Antonio, historical archives, musicians' petitions, musical cappella.

Sulla storia della basilica del Santo di Padova vi è una bibliografia davvero sterminata; le vicende del complesso conventuale sono state analizzate da diverse prospettive, in particolare quella storico-artistica, quella prevalentemente storica, quella religiosa e francescana, quella musicologica, nella maggior parte dei casi attingendo proprio alle fonti conservate nell'archivio storico della Veneranda Arca di Sant'Antonio. ${ }^{1}$

Ancora oggi però sono del tutto assenti gli studi sull'archivio nella sua interezza, sulla sua organizzazione e sulla storia istituzionale dell'ente che lo ha prodotto nell'arco di più di sei secoli di vita. Una mancanza questa, unita a motivazioni contingenti di tutela e di conservazione del patrimonio documentale, che ha spinto ad intraprendere un progetto di riordino e di inventariazione, tuttora in corso, per un'analisi approfondita "delle carte a partire dalle carte". ${ }^{2}$ L'obiettivo finale è non solo produrre uno strumento scientifica-

1 Foladore, "Basilica del Santo e Veneranda Arca," 489-497: 489-491.

2 Il progetto che ha visto la luce nel gennaio 2010 è tuttora in corso, è condotto da chi scrive con la supervisione scientifica di Giorgetta Bonfiglio-Dosio, già professore ordinario di Archivistica all'Università degli Studi di Padova, con la collaborazione del Centro Studi Antoniani (direttore padre Luciano Bertazzo), e della stessa Veneranda Arca. Il sostegno economico è garantito dalla 
mente valido, un inventario, che da una parte tuteli il patrimonio archivistico, dall'altra faciliti ed incentivi le ricerche degli studiosi, ma anche approfondire lo stretto legame tra la basilica del Santo e la Veneranda Arca, come queste due entità si sono rapportate tra loro sulla base della documentazione conservata in archivio.

Solamente per brevi cenni ricordo che tra la fine del XIV secolo e i primi decenni del XIX secolo il complesso conventuale del Santo era amministrato da due enti: da una parte, la comunità conventuale dei frati Minori per quanto attiene la regolamentazione della vita religiosa della comunità e lo svolgimento del culto antoniano, dall'altra, la Veneranda Arca di Sant'Antonio che amministrava il patrimonio in funzione della costituzione, abbellimento, manutenzione del complesso basilicale: entrambi hanno prodotto distintamente un loro archivio, in cui era custodita l'intera documentazione relativa alla conduzione dei loro affari. Diverse furono però le sorti delle-due istituzioni e, di riflesso, dei loro archivi.

Il convento del Santo fu coinvolto nella soppressione degli enti religiosi, decisa da Napoleone Bonaparte in due decreti del 28 luglio 1806 e del 25 aprile 1810: come i frati del Santo furono costretti ad abbandonare le loro stanze, così anche l'archivio del convento subì numerosi trasferimenti (e di conseguenza la perdita di parte del complesso documentale), fu indemaniato, al pari del restante patrimonio, ed è attualmente conservato dall'Archivio di Stato di Padova. ${ }^{3}$

L'archivio della Veneranda Arca invece scampò a queste disposizioni normative, restando fisicamente presso il soggetto produttore, poiché la maggioranza dei membri del collegio di Presidenza era laica e la stessa istituzione era considerata laica: questa peculiarità costituì la fortuna per la sopravvivenza pressoché integrale dell'archivio storico che documenta ancora oggi e ancora presso la basilica del Santo oltre 600 anni di storia del complesso conventuale e della città di Padova.

La Veneranda Arca di Sant'Antonio, un ente tuttora vivo e operante, ${ }^{4}$ è sostanzialmente una fabbriceria, un istituto di origini molto antiche. Le fabbricerie fondazionali sono istituti collegiali composti prevalentemente da laici, con il compito di amministrare il patrimonio delle chiese cattedrali o di monasteri di particolare rilevanza. La particolarità dell'Arca del Santo, rispetto alle altre fabbricerie in ambito italiano, è che essa non si riferisce alla chiesa cattedrale, ma amministra una chiesa "civica". ${ }^{5}$ I compiti di questo

Fondazione Cassa di Risparmio di Padova e Rovigo, nella persona del suo presidente Antonio Finotti.

3 Il fondo è attualmente conservato in Archivio di Stato di Padova (= ASPD), sezione III, Corporazioni religiose, Monasteri, Sant'Antonio; vedi MIBAC, Guida generale, 261.

4 Le disposizioni che regolano i compiti e l'amministrazione della Veneranda Arca sono contenuti nello Statuto approvato con regio decreto 11 marzo 1935, vedi Statuto della Veneranda Arca di Sant'Antonio di Padova, 1-6. Il collegio di Presidenza della Veneranda Arca è attualmente costituito dal padre Rettore della basilica, membro di diritto, da un esponente di nomina della Santa Sede, da cinque esponenti di nomina del Comune di Padova. I presidenti eleggono al loro interno a maggioranza un presidente capo e restano in carica per cinque anni, Statuto della Veneranda Arca di Sant'Antonio di Padova, 5, art. 5.

5 Per un intervento recente e puntuale rinvio a Bonfiglio Dosio, "L'archivio della Veneranda Arca," 221-230: 222-223. 
ente sono da sempre la tutela, la conservazione e la manutenzione dell'ingente patrimonio storico-artistico del complesso basilicale. ${ }^{6}$

"Entrando idealmente dentro l'archivio dell'Arca", i documenti più antichi che coprono con una sostanziale continuità i secoli XV-XIX in., costituiscono la sezione di antico regime, che comprende 1.102 unità archivistiche tra registri e buste, descritta sommariamente alla fine degli anni Venti del secolo scorso da Roberto Cessi. ${ }^{7}$

Nel corso della ricostruzione filologica della struttura originaria dell'archivio, l'analisi puntuale del materiale, condotta tramite il continuo raffronto con l'elenco sommario del Cessi, ha evidenziato che tale strumento descrittivo, finora a disposizione degli studiosi, è non solo sommario, ma in parecchi punti impreciso e inaffidabile in quanto non riconosce con precisione la tipologia dei documenti, i loro reciproci complessi legami, l'identità degli autori dei documenti e dei produttori delle serie archivistiche. Le sorprese non sono mancate: in molti casi si è constatata la manomissione successiva all'intervento del Cessi da parte di ordinatori e/o conservatori, spesso non meglio identificabili, che ha reso in talune circostanze inutilizzabile la sua descrizione.

Un esempio molto efficace in tal senso è dato proprio dalle suppliche dei musicisti della Cappella musicale del '700, le quali, sulla base dell'attuale riordino, costituiscono una sottoserie della serie Norme che regolano la vita e l'attività dell'Arca e della Cappella musicale. ${ }^{8}$

La serie, non originaria, in quanto creata da precedenti riordinatori con criteri non sempre condivisibili, comprende materiali abbastanza eterogenei, tutti però relativi alle norme che regolavano la vita dell'istituzione, con particolare riguardo alla Cappella musicale, ${ }^{9}$ attestata nell'archivio già a partire dal secolo XVI, e al concreto funzionamento di quest'ultima.

In questa serie sono state trovate solo le suppliche del secolo XVIII, mentre quelle precedenti sono incorporate nella serie Lettere e carteggio. Non è chiara la motivazione di questa suddivisione della medesima sottoserie, effettuata in passato: si può perfino supporre che le ricerche condotte su quel periodo storico abbiano spinto i conservatori dell'archivio a estrapolare le suppliche dei musici del Settecento dalle altre. Si può anche ipotizzare, ma con minor probabilità, che le suppliche allora 'più recenti' e - quindi - in fase di accettazione siano state tenute a parte e collegate con la normativa vigente, da poco risistemate in apposite raccolte.

Anche se non è stato possibile chiarire le ragioni di questa sistemazione pregressa e della separazione in due tronconi delle suppliche dei musici, si è assunta la tormentata

6 Statuto della Veneranda Arca di Sant’Antonio di Padova, 1, art. 3.

7 Vedi Cessi, Archivio Antico dell'Arca.

8 La serie copre un arco cronologico dal 1483 al 1812 ed è costituita da 27 unità archivistiche (23 registri e 4 cassette moderne di cartone).

9 Sulla Cappella musicale del Santo vedi Grossato, "Profilo culturale," 653-656; Sartori, "Documenti per la storia della musica;" Frasson, "La Cappella Musicale," 159-239; Storia della musica al Santo di Padova; Passadore, "Musicisti del Santo," 163-186; Dalla Vecchia, L'organizzazione della Cappella Musicale; Boscolo e Pietribiasi, La Cappella Musicale nel Settecento; Dalla Vecchia, "Tartini al Santo," 187-209; Padoan, "Musici al Santo," 757-788: 757-758, nota 1 con rimando a tutta la bibliografia precedente, specie per la Cappella musicale nei secoli XVI-XVII; Padoan, "L'armonico bombo," 23-45. 
decisione di non accorpare le suppliche settecentesche dei musicisti con quelle del Cinque e Seicento, conservate nella serie Lettere e carteggio: la ricostruzione organica della sottoserie delle suppliche dei musicisti avrebbe comportato sconvolgimenti troppo traumatici per l'archivio. Si è intervenuti solo in un caso: alcune suppliche del secolo XVIII, trovate disperse nell'archivio, sono state ricollocate nella serie Norme, segnalando però la posizione nella quale sono state rinvenute.

I precedenti interventi archivistici avevano sistemato le suppliche all'interno di alcuni fascicoli cartacei a loro volta collocati all'interno di due cassette moderne di cartone, seguendo l'ordine alfabetico dei cognomi dei musicisti, spesso confondendo persone diverse (padre e figlio, zio e nipote, nonno e nipote etc.): tale ordinamento, che si proponeva di realizzare uno strumento unico (elenco e indice) utile al rapido rinvenimento dei singoli personaggi, è stato ritenuto inidoneo alla ricostruzione dell'ordine originario dell'archivio e alla ricostruzione scientificamente fondata della vita della Cappella musicale.

Si è optato perciò per descrizioni analitiche a livello di unità documentale e si è pensato di ricorrere idealmente ad una sorta di griglia informativa, che risponda a due finalità: la prima, quella di agevolare gli studiosi che già a partire dall'elenco possono individuare dei nuclei tematici di ricerca e tramite l'indice dei nomi ritrovare con facilità le persone; la seconda, non meno importante, tutelare le carte, cercando di rallentare il lento processo di usura, determinato oltre che da fattori ambientali anche dal frequente "rimaneggiamento" della documentazione da parte degli utenti.

La descrizione analitica è così strutturata:

\begin{tabular}{|c|c|c|c|c|c|}
\hline $\begin{array}{c}\text { Tipologia } \\
\text { documentaria }\end{array}$ & $\begin{array}{c}\text { Nome e } \\
\text { cognome, } \\
\text { qualifica } \\
\text { professionale } \\
\text { del } \\
\text { richiedente }\end{array}$ & $\begin{array}{c}\text { Destinatario } \\
\text { della } \\
\text { richiesta }\end{array}$ & Motivazione & Data & $\begin{array}{c}\text { Descrizione } \\
\text { fisica e } \\
\text { presenza } \\
\text { di vecchie } \\
\text { segnature }\end{array}$ \\
\hline Supplica di & $\begin{array}{c}\text { Luca Zabile, } \\
\text { suonatore di } \\
\text { violino }\end{array}$ & $\begin{array}{c}\text { ai Presidenti } \\
\text { dell'Arca }\end{array}$ & $\begin{array}{c}\text { per ottenere } \\
\text { un aumento di } \\
\text { salario }\end{array}$ & (1716 dic. 24) & $\begin{array}{c}\text { c. sciolta } \\
\text { num. in } \\
\text { matita nel } \\
\text { presente } \\
\text { riordino } \\
\text { VS: } 128 / V \text { in } \\
\text { matita del sec. } \\
\text { XX }\end{array}$ \\
\hline
\end{tabular}

La schedatura analitica delle suppliche del secolo XVIII è stata effettuata da Giovanni Todesco, all'epoca laureando della laurea magistrale "Storia e gestione del patrimonio archivistico e bibliografico" interateneo Venezia-Padova..$^{10}$ Pertanto, dopo aver riunito in questa serie tutte le suppliche del secolo XVIII, anche quelle erroneamente lasciate in

${ }^{10}$ Giovanni Todesco, Musica al Santo: materiali archivistici relativi ai musici della Cappella, Corso di laurea magistrale interateneo Venezia-Ca' Foscari e Padova "Storia e gestione del patrimonio archivistico e bibliografico", a. acc. 2012-2013, relatore prof. Giorgetta Bonfiglio Dosio. 
precedenza in altre posizioni, si è scelto di disporle in ordine cronologico. In conseguenza di tale scelta la schedatura analitica per i documenti senza data e per l'identificazione delle persone si è avvalsa per i doverosi controlli, oltre che della consultazione di repertori specialistici, ${ }^{11}$ di riscontri puntuali sulle delibere della Presidenza che concedeva o negava quanto richiesto dal singolo musicista e ha consentito di rettificare alcune identificazioni errate e di distinguere parecchi omonimi, specie legati da vincoli di parentela. La data ricavata da altre fonti è indicata fra parentesi quadre. Nei casi in cui l'assenza di datazione della supplica e la consultazione di altre fonti non hanno consentito di reperire la delibera corrispondente si è indicato l'arco cronologico della carriera del musicista.

Vorrei illustrare ora in concreto alcune potenzialità che offre il nuovo riordino, estrapolate proprio dalla lettura dell'elenco che abbiamo realizzato.

I repertori specialistici già ricordati, si sono incentrati sull'organizzazione della Cappella musicale nel suo complesso, sulle dinamiche che regolavano il reclutamento e l'assunzione dei musicisti, le modalità di registrazione delle loro retribuzioni e delle eventuali sanzioni amministrative e pecuniarie (le cosiddette 'puntadure'); infine interessanti e utili approfondimenti sulla composizione dell'intero organico per ciascun anno.

Al di là di studi specifici su celeberrime figure di musici come per l'appunto Giuseppe Tartini, Francesco Antonio Vallotti per citare i più noti, sono scarse le indagini prosopografiche su singole figure di musici che si potrebbero incrementare con il nuovo riordino.

Un esempio è offerto da Domenico Dall'Oglio, violinista. Il volume curato da Boscolo e Pietribiasi non distingue in maniera corretta il musicista come anche i suoi fratelli Giuseppe, Antonio e Domenico, figlio di Antonio, creando una certa confusione, su cui è opportuno fare chiarezza. ${ }^{12}$

La famiglia Dall'Oglio ebbe come capostipite Domenico di professione liutaio, ${ }^{13}$ abitante nella parrocchia di San Lorenzo di Padova dove possedeva anche una bottega in affitto. Come documenta il censimento dell'8 febbraio 1720, Domenico, sposato con Pasqua, aveva a quell'epoca 5 figli: Domenico (di anni 11), Stefano (di anni 8), Giuseppe (di anni 6), Giacomo (di anni 3), Maddalena (al di sotto del primo anno di vita); non compare ancora Antonio, il quale pertanto fu il più giovane dei fratelli maschi e nacque quasi certamente dopo questo censimento. ${ }^{14}$

${ }^{11}$ In particolare i già citati Dalla Vecchia, "L'organizzazione della Cappella Musicale;" Boscolo e Pietribiasi, La Cappella Musicale Antoniana.

${ }^{12}$ Sono debitrice a Donato Gallo per la segnalazione e gli opportuni rinvii bibliografici.

${ }^{13}$ La sua attività di liutaio era molto nota e apprezzata al punto tale che gli valse il soprannome di Cannachionaro, come riferisce Gennari, Notizie giornaliere, I, 540.

${ }^{14}$ ASPD, sezione III, Censimenti, anagrafi e stato civile, b. 17, 1720, 8 febbraio. "Descrizione de gl'habitanti nella parrochia di San Lorenzo di Padova", vedi MIBAC, Guida generale, 255. Il documento è pubblicato in Facchin, "Costruttori di strumenti musicali," 202; è bene precisare che, nel ricostruire le vicende di questa famiglia, l'autore del saggio confonde Domenico padre, liutaio, con Domenico figlio, celebre violinista, infatti riferendosi al Domenico attestato nel censimento del 1720, scrive: "Questo celebre personaggio è noto soprattutto come violinista e solo marginalmente come liutaio, sebbene il documento lo censisca come tale" e poche righe più avanti: "Nel 1735 quando si recò in Russia, avrebbe dovuto avere circa 50 anni, quindi sarebbe tornato dalla Russia a circa 80 anni!.” È evidente che il "Domenico" censito nel 1720 è Domenico 
Ai tre figli, Domenico, Giuseppe e Antonio, Domenico padre fece seguire studi di musica con molta severità e fermezza, ${ }^{15}$ ottenendo per i primi due ottimi risultati: all'età di 18 anni, Domenico aveva già scritto alcuni concerti e dimostrava particolare abilità nel suonare il violino, mentre Giuseppe si dedicava al violoncello. ${ }^{16}$ Entrambi fecero parte dell'organico della Cappella musicale del Santo, nello specifico il 3 aprile 1732 Giuseppe Dall'Oglio presentava una supplica ai presidenti dell'Arca per essere assunto senza salario e con cotta. ${ }^{17}$

Nel 1735 Domenico Dall'Oglio, figlio, fu chiamato al servizio della corte russa come direttore dell'orchestra imperiale, partì con il fratello Giuseppe e lì rimasero per circa trent'anni, dove svolsero anche la funzione di mediatori per l'acquisto di quadri a Venezia e l'ingaggio di maestranze per i lavori nei palazzi imperiali. Tra il 1740 e il 1760 Domenico Dall’Oglio “quasi ogni anno commissionava a Padova e a Venezia un gran numero di soprapporte, per lo più paesaggi, rovine, e prospettive veneziane alla maniera di Canaletto, per rivenderle a famiglie nobili in gara tra di loro nell'allestire alla moda le proprie residenze". ${ }^{18}$ Il protrarsi del suo soggiorno moscovita si coglie molto bene in quattro suppliche indirizzate alla Presidenza dell'Arca che presentiamo, ${ }^{19}$ come anche il forte ruolo ricoperto dalla zarina, Anna I, nel prolungare la sua permanenza.

Nel 1765 i due fratelli Dall'Oglio ottennero l'autorizzazione per tornare in Italia: durante il viaggio, a Narva, Domenico fu colpito da apoplessia che lo condusse alla morte nel giro di pochi giorni. Giuseppe fu accolto presso la corte di Augusto III, re di Polonia, che in seguito lo nominò suo principale referente per trattare gli interessi della propria nazione presso la Repubblica di Venezia, una volta rientrato in patria. ${ }^{20}$

Per quanto riguarda Antonio Dall'Oglio, terzogenito di Domenico, liutaio, non dimostrando particolari doti nell'arte della musica, intraprese il commercio di pannilani e calzette. ${ }^{21}$ A sua volta ebbe un figlio, Domenico, anche lui suonatore di violino come il celebre zio, di cui si hanno scarne informazione biografiche: nella notte del 7 agosto 1789, all'età di 26 anni, fu costretto ad abbandonare la sua casa con Giuseppe Dalla Libera, cugino per ramo materno, e fuggire a Venezia. ${ }^{22}$

padre, di 35 anni, e non il figlio violinista, che aveva 11 anni all'epoca del censimento e quindi è possibile collocare la sua data di nascita attorno al 1709, pertanto il trasferimento presso la corte degli zar nel 1735 assieme al fratello Giuseppe, sarebbe avvenuto all'età di 26 anni, età plausibile come condizioni fisiche per affrontare un simile viaggio, ma soprattutto assolutamente compatibile con un percorso professionale già ben avviato e consolidato, come testimoniato dalle fonti.

15 "Ebbe tre figli e a tutti e tre fece insegnare la musica e a forza di bastone diventarono due infra loro eccellenti, uno nel violino e l'altro nel violoncello", vedi Gennari, Notizie giornaliere, I, 540.

${ }^{16}$ Pietrucci, Biografia degli artisti padovani, 93-94.

${ }^{17}$ Archivio storico della Veneranda Arca (= AArca), Serie 4, Norme che regolano la vita e l'attività dell'Arca e della Cappella musicale, (c.) 4.6 (127).3.67.

${ }^{18}$ Artemieva, "Della veneziana scuola," 19-58: 25.

${ }^{19}$ AArca, Serie 4, Norme che regolano la vita e l'attività dell'Arca e della Cappella musicale, (cc.) 4.6 (127).3.92; 4.7 (128).1.4; 4.7 (128).1.35; 4.7 (128).1.58.

${ }^{20}$ Pietrucci, Biografia degli artisti padovani, 94.

${ }^{21}$ Gennari, Notizie giornaliere, I, 540.

${ }^{22}$ Ibid., 539-540. 
Tornando più in generale alle potenzialità offerte dal riordino delle suppliche dei musicisti, si potrebbero anche compiere indagini quantitative mirate a verificare, ad esempio, sul totale delle suppliche censite quante riguardano la richiesta di aumento di salario oppure di una gratifica economica e quante, viceversa, chiedono una licenza.

Le suppliche dei musici possono essere una fonte preziosa anche per la storia del teatro, in particolare quello veneziano: ad esempio quella di Germano Pettenati, ${ }^{23}$ suonatore di violone, che il 27 novembre 1729 chiede ai presidenti dell'Arca di poter suonare al teatro di San Giovanni Grisostomo a Venezia, l'attuale teatro Malibran. La scheda di questo documento, e di molti altri analoghi, indica la duplice denominazione del teatro, sia quella in uso nel secolo XVIII, sia quella attuale, proprio per fornire un'informazione più completa agli studiosi.

Il dato fondamentale, che si evidenzia grazie al nuovo riordino, è l'immagine viva e nitida della Cappella musicale e della sua attività, la fitta rete di rapporti sociali che si intrecciavano, come emerge, ad esempio, nel caso concreto la richiesta di Antonio Loredan ${ }^{24}$ procuratore generale di Verona, stilata il 27 gennaio 1734, ai presidenti dell'Arca per avere presso di lui Filippo Lusi, ${ }^{25}$ suonatore di tromba, e una lettera dello stesso musicista, di un anno più tarda, che chiede di poterlo servire nuovamente.

Interessante è anche la supplica di Girolamo Soave, ${ }^{26}$ musico contralto, datata al 29 aprile 1726, non tanto per la sua richiesta di trattenersi fuori Padova per motivi di salute, quanto perché al documento è allegato il certificato medico del dottore Soardo Soardi di Brescia, autenticato dal notaio Enrico Bondiolus in data 21 marzo, attestante le sue precarie condizioni di salute.

Non mancano gli scorci sulla vita quotidiana dell'istituzione, come la supplica di padre Francesco Antonio Vallotti, ${ }^{27}$ maestro di Cappella, per far sostituire tutti i libri di musica a stampa ormai logori per il lungo e frequente utilizzo: la richiesta è priva di data, che è stata recuperata grazie ai doverosi controlli con la serie delle delibere della Presidenza (s.d. [1732 nov. $1^{\circ}$ ]).

Un altro esempio di supplica priva di data, a cui abbiamo attribuito comunque un arco cronologico di riferimento, è quella di Biagio Basson, ${ }^{28}$ organista della chiesa di Santa Maria del Carmine di Padova, in cui si rivolge ai presidenti dell'Arca per essere raccomandato all'elezione come primo organista al Santo (s.d. [post 1736 gen. 30 - ante 1736 mag. 19]).

È noto che molti musicisti, non secolari, avessero anche degli obblighi religiosi da rispettare, come ci conferma la supplica di padre Girolamo Valeriani di Antonio, ${ }^{29}$ suonatore di violoncello, ai presidenti dell'Arca per ottenere una licenza per predicare

${ }^{23}$ AArca, Serie 4, Norme che regolano la vita e l'attività dell'Arca e della Cappella musicale, (c.) 4.6 (127).3.39.

${ }^{24}$ Ibid., (c.) 4.6 (127).3.93.

${ }^{25}$ Ibid., (c.) 4.6 (127).3.97.

${ }^{26}$ Ibid., (c.) 4.6 (127).3.14.

${ }^{27}$ Ibid., (c.) 4.6 (127).3.73.

${ }^{28}$ Ibid., (c.) 4.6 (127).3.100.

${ }^{29}$ Ibid., (c.) 4.7 (128).1.15. 
durante la quaresima e far entrare il fratello Michele nell'organico della Cappella come sopranumerario (s.d. [post 1738 feb. 8 - ante 1739 dic. 29]).

Non di minore importanza sono anche le cosiddette suppliche "collettive", di cui sono rimasti pochi esempi. Eccone due: la prima, ${ }^{30}$ datata al 22 marzo 1727, in cui l'intero organico della Cappella fa richiesta all'Arca affinché appronti il consueto padiglione presso l'altare maggiore e fornisca le candele necessarie per le celebrazioni liturgiche in onore di santa Cecilia, loro protettrice, nel giorno della sua solennità (22 novembre); la seconda, ${ }^{31}$ datata al 4 marzo 1768, per ottenere un aumento di salario a causa dell'aumento generale del costo dei beni di sussistenza.

Non è possibile trovarsi a Pirano, sua città natale, e non accennare, almeno in chiusura, alla figura di Giuseppe Tartini. ${ }^{32}$ Del celebre violinista non si sono conservate suppliche, ma la sua presenza è comunque significativa nell'archivio della Veneranda Arca, a cominciare dalla delibera della Presidenza dell'Arca, con cui il 16 aprile 1721 si votava a favore della sua assunzione, fonte già edita da Boscolo e Pietribiasi, ma che è bene comunque richiamare in questa sede. Gli oltre quarant'anni di servizio del Tartini presso la Cappella musicale del Santo sono documentati nelle fonti contabili, soprattutto in corrispondenza del 31 dicembre di ciascun anno, quando i salariati e i musicisti venivano "reballottati" per l'anno successivo: a loro era corrisposto il salario pattuito, come documenta la registrazione dell'ultima retribuzione a Giuseppe Tartini il 31 dicembre 1769 nel giornale di cassa per l'anno relativo e nel corrispettivo mandato di pagamento, il $n^{\circ} 377$, scritto da Matteo Fanzago, cancelliere dell'Arca, su disposizione di Antonio Scipione Borromeo, presidente cassiere; sul verso della carta si legge la ricevuta dell'avvenuto pagamento autografa del Tartini. ${ }^{33}$ Soltanto due mesi dopo, il 26 febbraio 1770, egli morì e fu tumulato nella chiesa di Santa Caterina a Padova.

\section{Bibliografia}

Artemieva, Irina. "Della veneziana scuola siamo ricchi veramente.” In Capolavori nascosti dell'Ermitage. Dipinti veneti del Sei e Settecento da Pietroburgo, a cura di Irina Artemieva, Giuseppe Bergamini e Giuseppe Pavanello, 19-58. Milano: Electa, 1998.

Bonfiglio Dosio, Giorgetta. "L'archivio della Veneranda Arca di Sant'Antonio." In Cultura, arte e committenza al Santo nel Quattrocento. Atti del Convegno Internazionale di studi, Padova, 25-26 settembre 2009, a cura di Luciano Bertazzo e Giovanna Baldissin Molli, 221-230. Centro Studi Antoniani 43. Padova: Centro Studi Antoniani, 2010. Boscolo, Lucia e Pietribiasi, Maddalena, eds. La Cappella Musicale Antoniana di Padova nel secolo XVIII. Delibere della Veneranda Arca. Centro Studi Antoniani 29. Padova: Centro Studi Antoniani, 1997.

${ }^{30}$ Ibid., (c.) 4.7 (128).3.3.

${ }^{31}$ Ibid., (c.) 4.7 (128).4.9.

${ }^{32} \mathrm{Su}$ Giuseppe Tartini e la sua carriera all'ombra del Santo c'è una bibliografia davvero copiosa, mi limito a segnalare un recente lavoro, cui doverosamente rinvio per gli opportuni approfondimenti bibliografici: Dalla Vecchia, "Tartini al Santo," 187-209.

${ }^{33}$ AArca, Serie 15, Mandati filze, (b.) 15.215 (1052), n. 377. 
Cessi, Roberto. Archivio antico della Veneranda Arca di Sant’Antonio. Padova: Biblioteca Antoniana, [s.d.].

Dalla Vecchia, Jolanda. “Tartini al Santo.” In Giuseppe Tartini e la chiesa di Santa Caterina a Padova: archeologia, storia, arte intorno alla Chiesa di Santa Caterina d'Alessandria; risultati delle ricognizioni scientifiche delle tombe di Giuseppe Tartini, di Luigi Calza, dei parroci e degli studenti legisti, a cura di Vito Terribile Wiel Marin e Girolamo Zampieri, 187-209. Roma: L'Erma di Bretschneider, 1999.

____ L'organizzazione della Cappella Musicale Antoniana di Padova nel Settecento. Padova: Centro Studi Antoniani, 1995.

Facchin, Francesco. "Costruttori di strumenti musicali a Padova tra quattordicesimo e diciasettesimo secolo." In Botteghe artigiane dal Medioevo all'età moderna. Arti applicate e mestieri a Padova, a cura di Giovanna Baldissin Molli, 187-207. Padova, Il Prato, 2000.

Foladore, Giulia. "La basilica del Santo e la Veneranda Arca di Sant'Antonio nei documenti d'archivio: primi appunti.” Il Santo 52 (2012): 489-497.

Frasson, Leonardo. "La Cappella Musicale nella basilica del Santo e i suoi primi cinquant'anni di vita.” Il Santo 27 (1987): 159-239.

Gennari, Giuseppe. Notizie giornaliere di quanto avvenne specialmente in Padova dall'anno 1739 all'anno 1800, I-II, a cura di Loredana Olivato. Fossalta di Piave: Rebellato, 1982.

Grossato, Elisa. "Profilo culturale della cappella Musicale antoniana.” In Storia e cultura al Santo di Padova fra il XIII e il XX secolo, a cura di Antonino Poppi, 653-656. Vicenza, Neri Pozza, 1976.

Ministero per i beni culturali e ambientali - Ufficio centrale per i beni archivistici (=MIBAC), Guida generale degli Archivi di Stato italiani, III, Roma, Ministero, 1986, voce Padova.

Padoan, Maurizio. "L'armonico bombo: organici strumentali al Santo di Padova nel primo Barocco.” In Affetti musicali. Studi in onore di Sergio Martinotti, a cura di Maurizio Padoan, 23-45. Milano: Vita \& Pensiero, 2005.

____ . "Musici al Santo di Padova nel primo Barocco." In Florilegium Musicae. Studi in onore di Carolyn Gianturco, a cura di Patrizia Radicchi e Michael Burden 2, 757-788. Pisa: ETS, 2004.

Passadore, Francesco. "I musicisti del Santo e il mottetto a voce sola nel primo Seicento." Il Santo 32 (1992): 163-186.

Pietrucci, Napoleone. Biografia degli artisti padovani, Padova: Bianchi, 1858.

Sartori, Antonio. "Documenti per la storia della musica al Santo e nel Veneto." [A cura di Elisa Grossato; con un saggio di Giulio Cattin]. Vicenza: Neri Pozza, 1977.

Statuto della Veneranda Arca di Sant’Antonio di Padova, Padova: Tipografia del Messaggero, 1937.

Storia della musica al Santo di Padova, a cura di Sergio Durante e Pierluigi Petrobelli. Vicenza: Neri Pozza, 1990. 


\section{PROŠNJE GLASBENIKOV KAPELE SV. ANTONA PADOVANSKEGA \\ IZ 18. STOLETJA \\ PREUREDITEV IN MOŽNE RAZISKAVE ARHIVA USTANOVE \\ VENERANDA ARCA DI SANT’ANTONIO V PADOVI}

\section{Povzetek}

Veneranda Arca di S. Antonio je še vedno obstoječa in delujoča starodavna ustanova, ki je bila osnovana kot uprava (it. fabbriceria) cerkvenega premoženja. Posebnost te ustanove v primerjavi z ostalimi italijanskimi cerkvenimi uradi je v tem, da se ne nanaša na stolno cerkev, temveč na upravo »mestne« cerkve. Njene naloge so od nekdaj varovanje, ohranjanje in vzdrževanje obsežne kulturne dediščine bazilike sv. Antona.

$\mathrm{V}$ tej ustanovi se nahaja pomemben zgodovinski arhiv, ki še danes v prostorih nedaleč od bazilike sv. Antona hrani več kot 600 let staro zgodovino samostanskega kompleksa in mesta Padove. Od januarja leta 2010 v okviru štiriletnega projekta (pod vodstvom redne profesorice arhivistike na Univerzi v Padovi Giorgiette Bonfiglio Dosio) poteka preurejanje in popisovanje arhivskega inventarja, ki obsega več kot 250 tekočih metrov dokumentov. Cilj projekta je oblikovanje inventarja, ki bo po eni strani prispeval k varovanju dokumentov, po drugi pa ovrednotil dokumentarno zapuščino in nudil znanstveno orodje za nadaljnje raziskave.

Po teh kriterijih so prošnje glasbenikov, ki so v 18. stoletju delovali v glasbeni kapeli te ustanove, tudi preuredili. Za to ureditev ter analitične opise je zaslužen predvsem takrat podiplomski študent Giovanni Todesco, ki je na Univerzi Ca' Foscari v Benetkah in Univerzi v Padovi opravil nalogo z naslovom Zgodovina in upravljanje arhivske in bibliografske dediščine.

Pričujoča razprava na kratko povzema stanje pred novo ureditvijo, se dotakne zmot in dvomov, ki so se porajali med samim urejanjem in so na koncu pripeljali do nove ureditve prošenj. Obrazložen je ustroj nove oblike popisa, ki že po svoji naravi omogoča analitične opise, ki raziskovalcu prihranijo mnogo dela in časa. Na koncu so s pomočjo nekaj primerov prikazane možnosti in prednosti, ki jih ta ureditev omogoča pri novih raziskavah. 